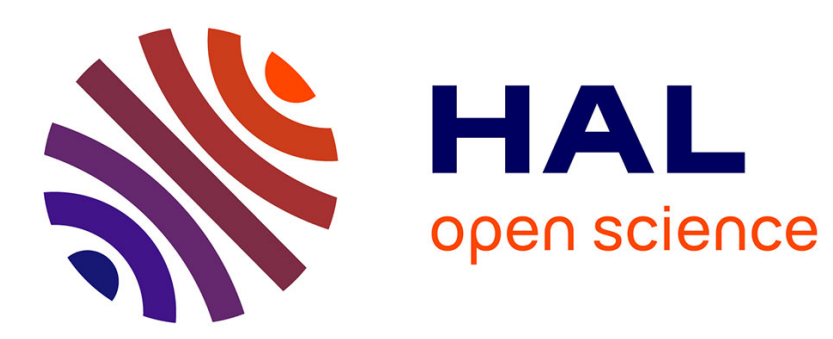

\title{
Results dealing with the behavior of the integrated density of states of random divergence operators
}

\author{
Hatem Najar
}

\section{To cite this version:}

Hatem Najar. Results dealing with the behavior of the integrated density of states of random divergence operators. 2005. hal-00007576v2

\section{HAL Id: hal-00007576 \\ https://hal.science/hal-00007576v2}

Preprint submitted on 22 Jul 2005

HAL is a multi-disciplinary open access archive for the deposit and dissemination of scientific research documents, whether they are published or not. The documents may come from teaching and research institutions in France or abroad, or from public or private research centers.
L'archive ouverte pluridisciplinaire HAL, est destinée au dépôt et à la diffusion de documents scientifiques de niveau recherche, publiés ou non, émanant des établissements d'enseignement et de recherche français ou étrangers, des laboratoires publics ou privés. 


\title{
Results dealing with the behavior of the integrated density of states of random divergence operators
}

\author{
25th July 2005
}

\section{Hatem Najar ${ }^{1}$}

\begin{abstract}
In this paper we generalize and improve results proven for acoustic operators in [9, 10]. It deals with the behavior of the integrated density of states of random divergence operators of the form $H_{\omega}=$ $\sum_{i, j=1}^{d} \partial_{x_{i}} a_{i, j}(\omega, x) \partial_{x_{j}}$; on the internal band edges of the spectrum. We propose an application of such a result to get localization.
\end{abstract}

2000 Mathematics Subject Classification :81Q10, 35P05, 37A30, 47F05.

Keywords and phrases :spectral theory, random operators, integrated density of states, Lifshitz tails, localization.

\section{Introduction}

Let us consider the random divergence operator

$$
H_{\omega}=-\nabla \rho_{\omega} \nabla=\sum_{i, j=1}^{d} \partial_{x_{i}} a_{i, j}(\omega, x) \partial_{x_{j}} ;
$$

\footnotetext{
${ }^{1}$ Département de Mathématiques Physiques I.P.E.I. Monastir, 5000 Monastir Tunisie . Researches partially supported by CMCU N 04/S1404 and Research Unity 01/UR/ 15-01 projects.
} 
where $\rho_{\omega}=\left(a_{i, j}(\omega, x)\right)_{1 \leq i, j \leq d}$ is an elliptic, $d \times d$-matrix valued, $\mathbb{Z}^{d}$-ergodic random field. i.e there exists some constant $\rho_{*}>1$, satisfying

$$
\frac{1}{\rho_{*}}|\xi|^{2} \leq\left\langle\rho_{\omega} \xi, \xi\right\rangle \leq \rho_{*}|\xi|^{2}, \quad \forall \xi \in \mathbb{C}^{d}
$$

This operator describes a vibrating membrane in the random medium as well as in the particular case when $\rho_{\omega}=\frac{1}{\varrho_{\omega}} \cdot I_{d},\left(I_{d}\right.$ is the identity matrix and $\varrho_{\omega}$ is a real function ) we get the acoustic operator [1, 9, 10]. The great interest of this operator both from the physical and the mathematical point of view is quite obvious and known [14].

As this paper is devoted to the study of the behavior of the integrated density of states, we start by recalling that it is defined as follows: We note by $H_{\omega, \Lambda}$ the restriction of $H_{\omega}$ to $\Lambda$ with self-adjoint boundary conditions. As $H_{\omega}$ is elliptic, the resolvent of $H_{\omega, \Lambda}$ is compact and, consequently, the spectrum of $H_{\omega, \Lambda}$ is discrete and is made of isolated eigenvalues of finite multiplicity [12]. We define

$$
N_{\Lambda}(E)=\frac{1}{\operatorname{vol}(\Lambda)} \cdot \#\left\{\text { eigenvalues of } \mathrm{H}_{\omega, \Lambda} \leq \mathrm{E}\right\}
$$

Here $\operatorname{vol}(\Lambda)$ is the volume of $\Lambda$ in the Lebesgue sense and \#E is the cardinal of $E$.

It is shown that the limit of $N_{\Lambda}(E)$ when $\Lambda$ tends to $\mathbb{R}^{d}$ exists almost surely and is independent of the boundary conditions. It is called the integrated density of states of $H_{\omega}$ (IDS as an acronym). See [4].

\subsection{The result}

The essential goal of this work is to study internal Lifshitz tails for the operator defined by (1.1). We review some results proven previously and improve them. In [9, 10 we have studied the same question under a special regime of disorder, precisely under the assumption that $\lim _{\varepsilon \rightarrow 0} \frac{\log \left|\log \mathbb{P}\left\{\omega_{0} \leq \varepsilon\right\}\right|}{\log \varepsilon}=0$. The main novelty here is that in the present work we omit this condition and we take a more general distribution of the random variables precisely we consider the case when $\log \mathbb{P}\left\{\omega_{0} \leq \varepsilon\right\} \sim-\varepsilon^{-\kappa}$ and we extend the result for another class of random Schrödinger operators.

As a possible application of our result we get localization near the band edges which is based on the fact that near those edges the integrated density of 
states exhibits Lifshitz tails. The main interest of such a technique lies in a much weaker assumption on the probability distribution.

\subsection{The model}

Assume that $\rho_{\omega}$ is of Anderson type i.e. it has the form

$$
\rho_{\omega}(x)=\rho^{+}(x)+\sum_{\gamma \in \mathbb{Z}^{d}} \omega_{\gamma} \rho^{0}(x-\gamma),
$$

where

(A.0)

- $\rho^{+}=\left(\rho_{i, j}^{+}\right)_{1 \leq i, j \leq d}$ is a, $\mathbb{Z}^{d}$-periodic and elliptic $d \times d$-matrix valued function.

- $\rho^{0}=\left(\rho_{i, j}^{0}\right)_{1 \leq i, j \leq d}$ is a $d \times d$-matrix valued function such that for some elliptic matrix, $\varrho_{+}^{0}$, we have

$$
0 \leq \sum_{\gamma \in \mathbb{Z}^{d}} \rho^{0}(x-\gamma) \leq \varrho_{+}^{0} .
$$

- $\left(\omega_{\gamma}\right)_{\gamma \in \mathbb{Z}^{d}}$ is a family of non constant and positive, independent identically distributed random variables taking values in $[0,1]$. We note by $(\Omega, \mathcal{F}, \mathbb{P})$ the probability space and we suppose that

$$
\lim _{\varepsilon \rightarrow 0^{+}} \frac{\log \left|\log \mathbb{P}\left(\left\{\omega_{0} \leq \varepsilon\right\}\right)\right|}{\log \varepsilon}=-\kappa, \quad \kappa \in[0,+\infty[.
$$

Let $h\left(\rho_{\omega}\right)$ be the quadratic form defined as follows: For $u \in H^{1}\left(\mathbb{R}^{d}\right)=$ $\mathcal{D}\left(h\left(\rho_{\omega}\right)\right)$

$$
\begin{aligned}
h\left(\rho_{\omega}\right)[u, u] & =\int_{\mathbb{R}^{d}} \rho_{\omega}(x) \nabla u(x) \cdot \overline{\nabla u(x)} d x \\
& =\sum_{1 \leq i, j \leq d} \int_{\mathbb{R}^{d}} a_{i, j}(\omega, x) \partial_{x_{i}} u(x) \overline{\partial_{x_{j}} u(x)} d x .
\end{aligned}
$$

$h\left(\rho_{\omega}\right)$ is a positive and closed quadratic form. $H_{\omega}$ given by (1.1) is defined as the self adjoint operator associated to $h\left(\rho_{\omega}\right)$ [12]. By this, $H_{\omega}$ is a measurable family of self adjoint and ergodic operators 11, 14. 


\subsection{Reference operator}

It is convenient to write $H_{\omega}$ as a perturbation of some background periodic operator $H_{0}$. More precisely we write:

$$
H_{\omega}=H_{0}+V_{\omega}
$$

with

$$
H_{0}=-\nabla \rho^{+} \nabla
$$

and

$$
V_{\omega}(\cdot)=-\nabla\left(\sum_{\gamma \in \mathbb{Z}^{d}} \omega_{\gamma} \rho^{0}(\cdot-\gamma)\right) \nabla \geq 0
$$

\subsubsection{Some facts from Floquet theory}

Now we review some standard facts from the Floquet theory for periodic operators. Basic references of this material can be found in [12].

As $\rho^{+}$is a $\mathbb{Z}^{d}$-periodic matrix, for any $\gamma \in \mathbb{Z}^{d}$, we have

$$
\tau_{\gamma} H_{0} \tau_{\gamma}^{*}=\tau_{\gamma} H_{0} \tau_{-\gamma}=H_{0}
$$

Let $\mathbb{T}^{*}=\mathbb{R}^{d} /\left(2 \pi \mathbb{Z}^{d}\right)$. We define $\mathcal{H}$ by

$\mathcal{H}=\left\{u(x, \theta) \in L_{l o c}^{2}\left(\mathbb{R}^{d}\right) \otimes L^{2}\left(\mathbb{T}^{*}\right) ; \forall(x, \theta, \gamma) \in \mathbb{R}^{d} \times \mathbb{T}^{*} \times \mathbb{Z}^{d} ; u(x+\gamma, \theta)=e^{i \gamma \theta} u(x, \theta)\right\}$.

There exists $U$ a unitary isometry from $L^{2}\left(\mathbb{R}^{d}\right)$ to $\mathcal{H}$ such that $H_{0}$ admits the Floquet decomposition [12]

$$
U H_{0} U^{*}=\int_{\mathbb{T}^{*}}^{\oplus} H_{0}(\theta) d \theta
$$

Here $H_{0}(\theta)$ is the operator $H_{0}$ acting on $\mathcal{H}_{\theta}$, defined by

$$
\mathcal{H}_{\theta}=\left\{u \in L_{l o c}^{2}\left(\mathbb{R}^{d}\right) ; \forall \gamma \in \mathbb{Z}^{d}, u(x+\gamma)=e^{i \gamma \theta} u(x)\right\} .
$$

As $H_{0}$ is elliptic, we know that, $H_{0}(\theta)$ has a compact resolvent; hence its spectrum is discrete [12]. We denote its eigenvalues, called Floquet eigenvalues of $H_{0}$, by

$$
E_{0}(\theta) \leq E_{1}(\theta) \leq \cdots \leq E_{n}(\theta) \leq \cdots
$$

The functions $\left(\theta \rightarrow E_{n}(\theta)\right)_{n \in \mathbb{N}}$ are Lipshitz-continuous, and we have

$$
E_{n}(\theta) \rightarrow+\infty \text { as } n \rightarrow+\infty \text { uniformly in } \theta \text {. }
$$


The spectrum $\sigma\left(H_{0}\right)$ of $H_{0}$ has a band structure. (i.e $\sigma\left(H_{0}\right)=\cup_{n \in \mathbb{N}} E_{n}\left(\mathbb{T}^{*}\right)$.) The periodic operator $H_{0}$ has an IDS which will be denoted by $n$. The behavior of $n$ at a band edge $E_{+}$, is said to be non-degenerate if,

$$
\lim _{\varepsilon \rightarrow 0^{+}} \frac{\log \left|n\left(E_{+}+\varepsilon\right)-n\left(E_{+}\right)\right|}{\log \varepsilon}=\frac{d}{2} .
$$

\subsubsection{The main assumptions}

As we study internal Lifshitz tails it is naturel to assume that $H_{0}$ has a spectral gap below $E_{+}$. More precisely we assume that:

\section{(A.1)}

There exists $E_{+}$and $\delta>0$ such that $\sigma\left(H_{0}\right) \cap\left[E_{+}, E_{+}+\delta\right)=\left[E_{+}, E_{+}+\delta\right)$ and $\sigma\left(H_{0}\right) \cap\left(E_{+}-\delta, E_{+}\right]=\emptyset$.

As, $V_{\omega} \geq 0$, the spectrum $\Sigma$ of $H_{\omega}$ contains an interval of the form $\left[E_{+}, E_{+}+\right.$ a] $(a>0)[3]$. As we are interested in the behavior of the IDS in the neighborhood of $E_{+}$, we require that $E_{+}$remains always the edge of a gap for $\Sigma$, when the perturbation is turned on. More precisely, if for all $t \in[0,1]$, we define $H_{\omega, t}=H_{0}+t V_{\omega}$ and $\Sigma_{t}$ is the almost sure spectrum of $H_{\omega, t}$, then one requires that the following assumption holds.

\section{(A.2)}

There exists $\delta^{\prime}>0$ such that for all $t \in[0,1], \Sigma_{t} \cap\left[E_{+}-\delta^{\prime}, E_{+}\right)=\emptyset$.

We assume also the following:

\section{(A.3)}

We will state that the behavior of the IDS depends on the form of the perturbation. One distinguishes between two behaviors of $\rho^{0}$.

Let $C_{0}=\left\{x \in \mathbb{R}^{d} ; \forall 1 \leq j \leq d ;-\frac{1}{2}<x_{j} \leq \frac{1}{2}\right\}$ and let $0<g_{-}<g_{+}$be two positives constants.

1. $\rho^{0}$ is of long range type.

There exists $\nu \in(d, d+2]$ such that for any $\gamma \in \mathbb{Z}^{d}, 1 \leq i, j \leq d$ and almost every $x$ in $C_{0}$ one has

$$
g_{-} \leq \rho_{i, j}^{0}(x-\gamma) \cdot(1+|\gamma|)^{\nu} \leq g_{+}
$$

and

$$
g_{-} \leq\left|\partial x_{i}\left(\rho_{i, j}^{0}\right)(x-\gamma)\right| \cdot(1+|\gamma|)^{\nu} \leq g_{+} .
$$

2. $\rho^{0}$ is of short range type.

There exists $\nu>d+2$ such that for any $\gamma \in \mathbb{Z}^{d}, 1 \leq i, j \leq d$ and almost 
every $x$ in $C_{0}$ one has

$$
0 \leq \rho_{i, j}^{0}(x-\gamma) \cdot(1+|\gamma|)^{\nu} \leq g_{+}
$$

and

$$
0 \leq\left|\partial x_{i}\left(\rho_{i, j}^{0}\right)(x-\gamma)\right| \cdot(1+|\gamma|)^{\nu} \leq g_{+}
$$

\section{Results and discussions}

The main result of this paper is the following:

Theorem 2.1 Let $H_{\omega}$ be the operator defined by (1.1). We assume that (A.1), (A.2) hold. Then if

1. $\rho^{0}$ is of long range type then,

$$
\begin{aligned}
\lim _{\varepsilon \rightarrow 0^{+}} \frac{\log \left(n\left(E_{+}+\varepsilon\right)-n\left(E_{+}\right)\right)}{\log \varepsilon}=\frac{d}{2} \Rightarrow \\
\quad \lim _{\varepsilon \rightarrow 0^{+}} \frac{\log \left|\log \left(N\left(E_{+}+\varepsilon\right)-N\left(E_{+}\right)\right)\right|}{\log \varepsilon}=-\sup \left(\frac{d}{2}+\kappa, \frac{d}{\nu-d}\right),
\end{aligned}
$$

if $\kappa+\frac{d}{2}<\frac{d}{\nu-d}$,

$$
\lim _{\varepsilon \rightarrow 0^{+}} \frac{\log \left|\log \left(N\left(E_{+}+\varepsilon\right)-N\left(E_{+}\right)\right)\right|}{\log \varepsilon}=-\frac{d}{\nu-d} .
$$

2. $\rho^{0}$ is of short range type then,

$$
\begin{aligned}
\lim _{\varepsilon \rightarrow 0^{+}} \frac{\log \left|\log \left(N\left(E_{+}+\varepsilon\right)-N\left(E_{+}\right)\right)\right|}{\log \varepsilon} & =-\left(\frac{d}{2}+\kappa\right) \Leftrightarrow \\
& \lim _{\varepsilon \rightarrow 0^{+}} \frac{\log \left(n\left(E_{+}+\varepsilon\right)-n\left(E_{+}\right)\right)}{\log \varepsilon}=\frac{d}{2} .
\end{aligned}
$$

Remark 2.2 The result of Theorem 2.1 is stated for lower band edges. Under adequate assumptions the corresponding result is true for upper band edges.

Now, let us comment the result. According to the Theorem 2.1 one notices that the behavior of the random variables is linked up to the lifshitz exponent, and determines if one is located in a classical regime or in a quantum one; 
i.e if the kinetic energy intervenes or if it does not in the Lifshitz exponent. In the long range case one sees that it depends on the value of $\kappa$, the Lifshitz asymptotics are classical (if $\kappa<\frac{d}{\nu-d}-\frac{d}{2}$ ) or quantum (if $\kappa>\frac{d}{\nu-d}-\frac{d}{2}$ ). In other terms in the case of the long range potential, Lifshitz exponent depends on the uncertainty principle, i.e on the kinetic energy only in the case when $\left(\frac{d}{\nu-d}<\kappa+\frac{d}{2}\right)$. In contrast, when $\left(\frac{d}{\nu-d}>\kappa+\frac{d}{2}\right)$ then the Lifshitz asymptotics are not governed by the same considerations. This is due to the fact that in the long range case as the potential decreases slowly, locally the potential is an empirical average of random variables. This leads to the fact that its effect is more important and more influencing than the spatial extension of the considered state.

From what it has been said previously, one concludes that the value of $\kappa$ is responsible for the transition between those two regimes.

The proof of the main result is now classic and based on the technic of periodic approximations which where originally stated by Klopp in [7]. It is quite close and follows the same steps used in [9, 10]. We omit details and we refer the reader to the above references.

\subsection{Application}

Now we state a useful result which can be related to the Theorem 2.1. Let

Theorem 2.3 Let $\theta \in \mathbb{R}^{d}$ and $E_{+}>0$ a band edge of the spectrum of $H_{\omega}$. Then for any $\alpha>1$, integer $p>0$, for $k \in \mathbb{N}$ sufficiently large, one has

$$
\text { (P1) } \mathbb{P}\left(\left\{\operatorname{dist}\left(\sigma\left(H_{\omega, \Lambda_{k} \alpha}^{\theta}\right), E_{+}\right) \leq \frac{1}{k}\right\}\right) \leq \frac{1}{k^{p}} .
$$

Where $\Lambda_{k}$ is the box centered in 0 of side length $2 k+1$ and $A_{\omega, \Lambda_{k}}^{\theta}$ is the operator $H_{\omega}$ restricted to this box with $\theta$-quasiperiodic boundary condition i.e with boundary condition $\varphi(x+\gamma)=e^{i \gamma \cdot \theta} \varphi(x)$ for any $\gamma \in(2 k+1) \mathbb{Z}^{d}$.

To be able to apply the multiscale analysis [1, 14], we assume that $\rho^{0}$ is compactly supported. Indeed, when the single site is compactly supported $H_{\omega}$ satisfies a Wegner estimate [1] i.e for some $\alpha>0$ and $n>0$ for $E \in \mathbb{R}$ for $k \geq 1$ and $0<\varepsilon<1$, there exists $C(E)>0$ such that one has

$$
\text { (P2) } \mathbb{P}\left(\left\{\operatorname{dist}\left(\sigma\left(H_{\omega, \Lambda_{k}}^{\theta}\right), E\right) \leq \varepsilon\right\}\right) \leq C(E) \cdot\left|\Lambda_{k}\right|^{\alpha} \cdot \varepsilon^{n} .
$$

So, for a band edge energy $E_{+}$using the Theorem 2.3 for $\theta=0$, we obtain the initial estimate to start a multi-scale analysis. This proves that the spectrum 
of $H_{\omega}$ is exponentially localized in some interval around the energy $E_{+}$i.e that in some neighborhood of $E_{+}$eigenfunctions associated to energies in that interval are exponentially localized. More precisely we have

Theorem 2.4 Let $H_{\omega}$ defined by (1.1). We assume that (A.1) and (A.2) hold and the single site is compactly supported. There exists $\varepsilon_{0}>0$ such that (i) $\Sigma \cap\left[E_{+}, E_{+}+\varepsilon_{0}\right]=\Sigma_{p p} \cap\left[E_{+}, E_{+}+\varepsilon_{0}\right]$.

(ii) an eigenfunction corresponding to an eigenvalue in $\left[E_{+}, E_{+}+\varepsilon_{0}\right]$ decays exponentially.

(iii) for all $p>0$,

$$
\mathbb{E}\left\{\left.\sup _{t>0}\|\| X\right|^{p} e^{i t H_{\omega}} P_{\left[E_{+}, E_{+}+\varepsilon_{0}\right]}\left(H_{\omega}\right) \chi_{K} \|\right\}<+\infty .
$$

Here $P_{I}\left(H_{\omega}\right)$ is the spectral projection on the interval $I, \chi_{K}$ is the characteristic function of $K, K$ is a compact of $\mathbb{R}^{d}$ and $X$ is the position operator.

To comment upon Theorem 2.4, let us consider the wave equation:

$$
\frac{\partial^{2} u}{\partial t^{2}}=H_{\omega} u
$$

The solution of (2.13) is given [14 by

$$
u(t, \cdot)=\cos \left(t \sqrt{H_{\omega}}\right) u_{0}+\sin \left(t \sqrt{H_{\omega}}\right) u_{1},
$$

where $u_{0}=u(0, \cdot)$ and $\sqrt{H_{\omega}} u_{1}=\left(\partial_{t} u\right)(0, \cdot)$ denote the initial data.

The result of Theorem 2.3 and the one of Theorem 2.4 can be related to the behavior of the integrated density of states in the neighborhood of the socalled fluctuation boundary $E_{+}$[6, 11]. This is done in the Schrödinger case in 15 .

\subsection{The periodic approximations}

Let us consider the following periodic operator

$$
H_{\omega, k}=-\nabla \cdot \rho_{\omega, k} \cdot \nabla,
$$

where $\rho_{\omega, k}$ is the following matrix

$$
\rho_{\omega, k}=\rho^{+}+\sum_{\gamma \in C_{k} \cap \mathbb{Z}^{d}} \omega_{\gamma} \sum_{\beta \in(2 k+1) \mathbb{Z}^{d}} \rho^{0}(\cdot-\gamma-\beta) .
$$


$C_{k}$ is the cube

$$
C_{k}=\left\{x \in \mathbb{R}^{d} ; \forall 1 \leq j \leq d,-\frac{2 k+1}{2}<x_{j} \leq \frac{2 k+1}{2}\right\} .
$$

We set

$$
V_{\omega, k}=-\nabla \cdot\left(\sum_{\gamma \in C_{k} \cap \mathbb{Z}^{d}} \omega_{\gamma} \sum_{\beta \in(2 k+1) \mathbb{Z}^{d}} \rho^{0}(\cdot-\gamma-\beta)\right) \cdot \nabla
$$

$H_{\omega, k}$ is $(2 k+1) \mathbb{Z}^{d}$-periodic and essentially self adjoint operator. Let $\mathbb{T}_{k}^{*}=$ $\left(\mathbb{R}^{d}\right) / 2(2 k+1) \pi \mathbb{Z}^{d}$. We define $N_{\omega, k}$ the IDS of $H_{\omega, k}$ by

$$
N_{\omega, k}(E)=\frac{1}{(2 \pi)^{d}} \sum_{n \in \mathbb{N}} \int_{\left\{\theta \in \mathbb{T}_{k}^{*}, E_{\omega, k, n}(\theta) \leq E\right\}} d \theta .
$$

Let $d N_{\omega, k}$ be the derivative of $N_{\omega, k}$ in the distribution sense. As $N_{\omega, k}$ is increasing, $d N_{\omega, k}$ is a positive measure; it is the density of states of $H_{\omega, k}$. We denote by $d N$ the density of states of $H_{\omega}$.

Theorem 2.5 [5, 9] For any $\varphi \in \Lambda_{0}^{\infty}(\mathbb{R})$ and for almost all $\omega \in \Omega$ we have

$$
\lim _{k \rightarrow \infty}\left\langle\varphi, d N_{\omega, k}\right\rangle=\langle\varphi, d N\rangle .
$$

In what follows we give a well-known [7, 9, 10] result stating that the IDS of $H_{\omega}$ is exponentially well approximated by the expectation of the IDS of the periodic operators $H_{\omega, k}$ when $k$ is polynomial in $\varepsilon^{-1}$. More precisely

Lemma 2.6 For any $\eta_{0}>1$ and $I \subset \mathbb{R}$ a compact interval, there exists $\nu_{0}>0$ and $\varepsilon_{0}>0$ such that, for $0<\varepsilon<\varepsilon_{0}, E \in I$ and $k \geq k_{1}=\varepsilon^{-\nu_{0}}$, we have

$$
\begin{aligned}
\mathbb{E}\left[N_{\omega, k}(E+\varepsilon / 2)-N_{\omega, k}(E-\varepsilon / 2)\right]-e^{\varepsilon^{-\eta_{0}}} & \\
& \leq N(E+\varepsilon)-N(E) \\
\leq & \mathbb{E}\left[N_{\omega, k}(E+2 \varepsilon)-N_{\omega, k}(E-2 \varepsilon)\right]+e^{-\varepsilon^{-\eta_{0}}}
\end{aligned}
$$

\section{The proof of Theorem 2.1}

To prove Theorem 2.1, we use periodic approximations. We prove a lower and an upper bounds on $N\left(E_{+}+\varepsilon\right)-N\left(E_{+}\right)$. The upper and lower bounds are proven separately. 


\subsection{The lower bound}

We postpone the proof of the lower bound. More details can be found in [9, 10. It consists in proving the following theorem.

Theorem 3.1 Let $H_{\omega}$ be the operator defined by (1.1). We assume that (A.1), (A.2) hold. Then,

- if $\rho^{0}$ is of long range type, we have

$$
\liminf _{\varepsilon \rightarrow 0^{+}} \frac{\log \left|\log \left(N\left(E_{+}+\varepsilon\right)-N\left(E_{+}\right)\right)\right|}{\log \varepsilon} \geq-\sup \left(\frac{d}{2}+\kappa, \frac{d}{\nu-2}\right) .
$$

- if $\rho^{0}$ is of short range type, we have

$$
\liminf _{\varepsilon \rightarrow 0^{+}} \frac{\log \left|\log \left(N\left(E_{+}+\varepsilon\right)-N\left(E_{+}\right)\right)\right|}{\log \varepsilon} \geq-\left(s \frac{d}{2}+\kappa\right) .
$$

Here $s<1$ if $n$ is degenerate and $s=1$ if not.

Proof. By assumption, there is a spectral gap below $E_{+}$of length at least $\delta^{\prime}>0$. Thus, for $\varepsilon<\delta^{\prime}$ we have

$$
N\left(E_{+}+\varepsilon\right)-N\left(E_{+}\right)=N\left(E_{+}+\varepsilon\right)-N\left(E_{+}-\varepsilon\right) .
$$

To prove Theorem 3.1, it suffice to lower bound $N\left(E_{+}+\varepsilon\right)-N\left(E_{+}-\varepsilon\right)$. Then, for $N$ large, we will show that $H_{\omega, \Lambda_{N}}\left(H_{\omega, \Lambda_{N}}\right.$ is $H_{\omega}$ restricted to $\Lambda_{N}$ with Dirichlet boundary conditions) has a large number of eigenvalues in $\left[E_{+}-\varepsilon, E_{+}+\varepsilon\right]$ with a large probability. For this we will construct a family of approximate eigenvectors associated to approximate eigenvalues of $H_{\omega, \Lambda_{N}}$ in $\left[E_{+}-\varepsilon, E_{+}+\varepsilon\right]$. These functions can be constructed from an eigenvector of $H_{0}$ associated with $E_{+}$. Locating this eigenvector in $\theta$ and imposing to $\omega_{\gamma}$ to be small for $\gamma$ in some well chosen cube, one obtains an approximate eigenfunction of $H_{\omega, \Lambda_{N}}$. Locating the eigenfunction in $x$ in several disjointed places, we get several eigenfunctions two by two orthogonal. The subtlety is in the good choice of the size of the cube.

Using the same computation done in [9, 10] we get that we have to estimate the following two probabilities: For $1>\alpha>0$,

$$
\mathbb{P}_{\varepsilon, \alpha, 1}=\mathbb{P}\left(\left\{\omega ;|\beta| \leq \varepsilon^{-(1+\alpha) / 2} ; \sum_{\gamma \in \mathbb{Z}^{d}} \omega_{\gamma}(1+|\beta-\gamma|)^{-\nu} \leq \varepsilon^{1+\alpha}\right\}\right)
$$




$$
\mathbb{P}_{\varepsilon, \alpha, 2}=\mathbb{P}\left(\left\{\omega ; \sum_{\gamma \in \Lambda_{\alpha}\left(\varepsilon^{s}\right)} \omega_{\gamma}(1+|\gamma|)^{-\nu} \leq \frac{\varepsilon^{1+\alpha}}{2}\right\}\right)
$$

here $\Lambda_{\alpha}(\zeta)=\left\{\gamma \in \mathbb{Z}^{d} ; \forall 1 \leq j \leq d ;\left|\gamma_{j}\right| \leq \zeta^{-\left(\frac{1}{2}+\alpha\right)}\right\}$.

Indeed we have the following relations [9, 10]:

- if $\rho^{0}$ is of long range type, we have

$$
\liminf _{\varepsilon \rightarrow 0^{+}} \frac{\log \left|\log \left(N\left(E_{+}+\varepsilon\right)-N\left(E_{+}\right)\right)\right|}{\log \varepsilon} \geq \liminf _{\varepsilon \rightarrow 0^{+}} \frac{\log \left|\log \left(\mathbb{P}_{\varepsilon, \alpha, 1}\right)\right|}{\log \varepsilon} .
$$

- if $\rho^{0}$ is of short range type, we have

$$
\liminf _{\varepsilon \rightarrow 0^{+}} \frac{\log \left|\log \left(N\left(E_{+}+\varepsilon\right)-N\left(E_{+}\right)\right)\right|}{\log \varepsilon} \geq \liminf _{\varepsilon \rightarrow 0^{+}} \frac{\log \left|\log \left(\mathbb{P}_{\varepsilon, \alpha, 2}\right)\right|}{\log \varepsilon} .
$$

Now one deals with the estimation of $\mathbb{P}_{\varepsilon, \alpha, 1}$ and $\mathbb{P}_{\varepsilon, \alpha, 2}$. We start by:

- The estimation of $\mathbb{P}_{\varepsilon, \alpha, 1}$.

Let $\nu \in(d, d+2]$. First we notice that if

$$
\omega_{\gamma} \leq \varepsilon^{1+\alpha} \text { for }|\gamma| \leq \varepsilon^{-(1-\alpha) / 2}
$$

and

$$
\omega_{\gamma} \leq \varepsilon^{1+\alpha}\left(1+\operatorname{dist}\left(\gamma, C_{0, \varepsilon^{-(1-\alpha) / 2}}\right)\right)^{(\nu-d)(1-\alpha)} \text { for } \varepsilon^{-(1-\alpha) / 2}<|\gamma| \leq \varepsilon^{-\frac{1+2 \alpha}{\nu-d}},
$$

then

$$
\sum_{\gamma \in \mathbb{Z}^{d}} \omega_{\gamma}(1+|\beta-\gamma|)^{-\nu} \leq \varepsilon^{1+\alpha}
$$

So

$$
\mathbb{P}_{\varepsilon, \alpha, 1} \geq \mathbb{P}_{2} \cdot \mathbb{P}_{1}
$$

Where

$$
\mathbb{P}_{1}=\mathbb{P}\left\{\omega ; \forall \gamma \text { such that }|\gamma| \leq \varepsilon^{-(1-\alpha) / 2}, \omega_{\gamma} \leq \varepsilon^{1+\alpha}\right\}
$$

and

$$
\begin{aligned}
\mathbb{P}_{2}=\mathbb{P}\left\{\omega ; \forall \gamma \text { such that } \varepsilon^{-(1-\alpha) / 2}<|\gamma| \leq \varepsilon^{-\frac{1+2 \alpha}{\nu-d}},\right. \\
\left.\omega_{\gamma} \leq \varepsilon^{1+\alpha}\left(1+\operatorname{dist}\left(\gamma, C_{0, \varepsilon^{-(1-\alpha) / 2}}\right)\right)^{(\nu-d)(1-\alpha)}\right\} .
\end{aligned}
$$


As the random variables are i.i.d we get that

$$
\mathbb{P}_{1}=\left(\mathbb{P}\left\{\omega_{0} \leq \varepsilon^{1+\alpha}\right\}\right)^{\varepsilon^{-d(1-\alpha) / 2}}
$$

and

$$
\left.\mathbb{P}_{2}=\prod_{\substack{\varepsilon^{(1-\alpha) / 2}<|\gamma| \leq \varepsilon^{-\frac{1+2 \alpha}{\nu-d}}}} \mathbb{P}\left(\omega_{0} \leq \varepsilon^{1+\alpha}\left(1+\operatorname{dist}\left(\gamma, C_{0, \varepsilon^{-(1-\alpha) / 2}}\right)\right)^{(\nu-d)(1-\alpha)}\right)\right) .
$$

Now by applaying the logarithm to (3.20) and taking into akount (3.21) and (3.22) wile using (1.5), for $\alpha$ and $\varepsilon$ small enough we get that

$$
\begin{aligned}
& \log \mathbb{P}_{\varepsilon, \alpha, 1} \geq \\
& -\varepsilon^{-(\kappa+d / 2)(1+\alpha)}-\varepsilon^{-\kappa(1+\alpha)} \sum_{\varepsilon^{-(1-\alpha) / 2} \leq|\gamma| \leq \varepsilon^{-\frac{1+2 \alpha}{\nu-d}}}\left(1+\operatorname{dist}\left(\gamma, C_{0, \varepsilon^{-(1-\alpha) / 2}}\right)\right)^{-\kappa(\nu-d)(1-\alpha)} .
\end{aligned}
$$

As if $(\nu-d) \kappa>d$ the sum in (3.23) converges when $\alpha$ is chosen small enough such that $(1-\alpha)(\nu-d) \kappa>d$. So we get,

$$
\liminf _{\varepsilon \rightarrow 0^{+}} \frac{\log \left|\log \left(\mathbb{P}_{\varepsilon, \alpha, 2}\right)\right|}{\log \varepsilon} \geq-(1+\alpha)\left(\kappa+\frac{d}{2}\right) .
$$

In the case when $(\nu-d) \kappa<d$, for $\varepsilon$ small one computes the sum in (3.23) we get the following estimation

$$
\begin{gathered}
\sum_{\varepsilon^{-(1-\alpha) / 2} \leq|\gamma| \leq \varepsilon^{-\frac{(1+2 \alpha)}{(\nu-d)}}}\left(1+\operatorname{dist}\left(\gamma, C_{0}, \varepsilon^{-(1-\alpha) / 2}\right)\right)^{-\kappa(\nu-d)(1-\alpha)} \leq \\
C \cdot \varepsilon^{\kappa(1-\alpha)} \cdot \varepsilon^{-d(1+\alpha) /(\nu-d)} .
\end{gathered}
$$

Using equations (3.23) and (3.25) and the fact that $\frac{d}{\nu-d}-\kappa+\frac{d}{2} \geq 0$ we get

$$
\log \mathbb{P}_{\varepsilon, \alpha, 1} \geq-C \varepsilon^{-(\kappa+d / 2)(1+\alpha)} \cdot \varepsilon^{-(d(1+\alpha)-2 \alpha \kappa) /(\nu-d)} .
$$

We apply the logarithm into the last equation taking into a count (1.5), (3.18) and (3.24) we get (3.16). 
- The estimation of $\mathbb{P}_{\varepsilon, \alpha, 2}$. Let us notice that there exists $C>0$ such that we have

$$
\mathbb{P}_{\varepsilon, \alpha, 2} \geq \mathbb{P}\left\{\omega ; \forall \gamma \in \Lambda_{\alpha}\left(\varepsilon^{s}\right) ; \omega_{\gamma} \leq \frac{\varepsilon^{1+\alpha}}{C}\right\} .
$$

As the random variables are i.i.d we get that

$$
\mathbb{P}_{\varepsilon, \alpha, 2} \geq \Pi_{\gamma \in \Lambda_{\alpha}\left(\varepsilon^{s}\right)} \mathbb{P}\left\{\omega ; \omega_{\gamma} \leq \frac{\varepsilon^{1+\alpha}}{C}\right\}=\left(\mathbb{P}\left\{\omega_{0} \leq \frac{\varepsilon^{1+\alpha}}{C}\right\}\right)^{\sharp \Lambda_{\alpha}\left(\varepsilon^{s}\right)}
$$

Now taking into account (1.5), (3.19), the fact that $\sharp \Lambda_{\alpha}(\varepsilon)=\varepsilon^{-d\left(\frac{1}{2}+\alpha\right)}$, and $\alpha>0$ small we end the proof of (3.17). So the proof of Theorem 3.1 is ended.

\subsection{The upper bound}

To prove the upper bound, we compare $N\left(E_{+}+\varepsilon\right)-N(E)$ to the IDS some reduced operators. More precisely, we prove that for an energy $E$ close to $E_{+}, N(E)-N\left(E_{+}\right)$can be upper bounded by the IDS of some random and bounded operator. Indeed we have,

Lemma 3.2 g/ Let $H_{\omega}$ be the operator defined by (1.1). We assume that (A.1), (A.2) and (A.3) hold. There exists $E_{0}>E_{+}$and $C>1$ such that, for $E_{+} \leq E \leq E_{0}$ we have

$$
0 \leq N(E)-N\left(E_{+}\right) \leq N_{\mathcal{E}_{0}}\left(C \cdot\left(E-E_{+}\right)+E_{+}\right)
$$

where $N_{\mathcal{E}_{0}}$ is the IDS of $H_{\omega}^{0}=\Pi_{0} H_{\omega} \Pi_{0}$ and $\Pi_{0}$, is the spectral projection for $H_{0}$ on the band starting at $E_{+}$.

Proof: The proof of Lemma 3.2 is given in the case of acoustic operators in [9]. It is still true in the divergence case. It is based on a localization in energy for the density of states. It goes as follows: We approach the density of states of $H_{\omega}$ by the density of states of periodic approximations, see section 2.2. In a neighborhood of $E_{+}$, we control the behavior of the density of states of periodic approximations via the density of states of periodic approximations of the reference operator i.e $H_{\omega}^{0}=H_{0}^{0}+V_{\omega}^{0}$. We then compute the limit for the density of states of the reference operators and we obtain the sought for result. 


\subsubsection{The short range case}

We recall that in this case we assume that the IDS, $n$ of the background operator $H_{0}$ is non-degenerate. We prove the following theorem:

Theorem 3.3 Let $H_{\omega}$ be the operator defined by (1.1). We assume that (A.1) and (A.2) hold and $n$ is non-degenerate at $E_{+}$, then

$$
\limsup _{\varepsilon \rightarrow 0^{+}} \frac{\log \left|\log \left(N\left(E_{+}+\varepsilon\right)-N\left(E_{+}\right)\right)\right|}{\log \varepsilon} \leq-\left(\frac{d}{2}+\kappa\right) .
$$

Proof: Let us notice that by Lemma 3.2 to prove Theorem 3.3 it suffices to get the same upper bound for the reference operator. This represents several advantages: first, $H_{\omega}^{0}$ it is a bounded random operator and equivalent to a random Jacobi matrix acting on $L^{d}\left(\mathbb{T}^{*}\right) \otimes \mathbb{C}^{n_{0}}$ (Here $n_{0}$ is the number of Floquet eigenvalues generating the band starting in $E_{+}$). The second advantage is that while, $E_{+}$is an interior edge of a gap for $H_{\omega}$, it becomes the bottom of the spectrum for $H_{\omega}^{0}$.

The idea of the proof is based on the uncertainly principle. Indeed, as $V_{\omega}^{0} \geq 0$, if a vector minimizes $H_{\omega}^{0}$, it necessarily minimizes $H_{0}^{0}=\Pi_{0} H_{0} \Pi_{0}$; hence, it has to be concentrated in the quasimomentum $\theta$ near the zeros of $\left(E_{j}(\theta)-\right.$ $\left.E_{+}\right)_{1 \leq j \leq n_{0}}$. For this, we have to take into account all the points where the Floquet eigenvalues reach $E_{+}$. Let $\theta^{0}$ be one of those points. As $n$ is nondegenerate, for $c>0$ small, in a neighborhood of $\theta^{0}$ we have [9]

$$
\mathbb{D}=c \sum_{j=1}^{d}\left(1-\cos \left(\theta_{j}-\theta_{j}^{0}\right)\right) \leq H_{0}^{0}-E_{+} \cdot I_{d} .
$$

Here $\mathbb{D}$ is acting on $L^{2}\left(\mathbb{T}^{*}\right) \otimes \mathbb{C}^{n_{0}}$ and $I_{d}$ is the identity matrix.

We recall that $V_{\omega}$ is the operator defined by

$$
V_{\omega}=-\nabla\left(\sum_{\gamma \in \mathbb{Z}^{d}} \omega_{\gamma} \rho^{0}(\cdot-\gamma)\right) \nabla .
$$

It is proved [9] that $V_{\omega}^{0}$ can be lower bounded by

$$
V_{2, \omega}^{a}=\sum_{\gamma \in \mathbb{Z}^{d}} \omega_{\gamma} \Pi_{\gamma} .
$$


Here $\Pi_{\gamma}$ is the orthogonal projection on the vector $\theta \mapsto e^{i \gamma \theta}$ in $L^{2}\left(\mathbb{T}^{*}\right) \otimes \mathbb{C}^{n_{0}}$. Now using the following (unitary operator) discrete Fourier transformation defined from $l^{2}\left(\mathbb{Z}^{d}\right)$ to $L^{2}\left([0,2 \pi]^{d}\right)$ by

$$
\mathcal{F}(u)(k)=\widehat{u}(k)=\sum_{n \in \mathbb{Z}} u(n) e^{-i n \cdot k}
$$

we get that $\mathbb{D}$ is unitarly equivalent to the usual discrete Schrödinger operator. So $H_{\omega}^{0}$ is lower bounded by some opertaor which it self unitarly equivalent to the usual discrete random operator whose behavior of the IDS at the edges of the spectral gaps is already known [5, 9]. This lower bound on the operator immediately yields an upper bound on the density of states.

\subsubsection{The long range case}

In this section we shale prove:

Theorem 3.4 Let $H_{\omega}$ be the operator defined by (1.1). We assume that (A.1), (A.2) hold. If $\frac{d}{\nu-d}>\kappa+\frac{d}{2}$ then,

$$
\limsup _{\varepsilon \rightarrow 0^{+}} \frac{\log \left|\log \left(N\left(E_{+}+\varepsilon\right)-N\left(E_{+}\right)\right)\right|}{\log \varepsilon} \leq-\frac{d}{\nu-d} .
$$

If $n$, the IDS of $H_{0}$ is non-degenerate then,

$$
\limsup _{\varepsilon \rightarrow 0^{+}} \frac{\log \left|\log \left(N\left(E_{+}+\varepsilon\right)-N\left(E_{+}\right)\right)\right|}{\log \varepsilon} \leq-\sup \left(\frac{d}{2}+\kappa, \frac{d}{\nu-d}\right) .
$$

\section{Proof:}

- If $\frac{d}{\nu-d}>\kappa+\frac{d}{2}$.

Notice that in this case we have no assumption made on the behavior of $n$, the IDS of the periodic operator. The proof goes exactly as the one given in [10], for this we omit details. From Lemma 2.6 and for $\eta_{0}>1 /(\nu-d)$ and $k \sim \varepsilon^{-\delta}$ such that $\delta>\nu_{0}$ the proof of (3.29) is reduced to prove that

$$
\limsup _{\varepsilon \rightarrow 0^{+}} \frac{\log \left|\log \left(\mathbb{E}\left(N_{\omega, k}\left(E_{+}+\varepsilon\right)-N_{\omega, k}\left(E_{+}\right)\right)\right)\right|}{\log \varepsilon} \leq-\frac{d}{\nu-d} .
$$


Lemma 3.5 $9 /$ Let $k \sim \varepsilon^{-\rho}$ with $\rho>1 /(\nu-d)$. Define the event,

$$
\mathbf{E}_{\varepsilon, \omega}=\left\{\omega ; V_{\omega, k} \geq-\varepsilon \Delta=-\varepsilon \sum_{i=1}^{d} \partial_{x_{i}}^{2}\right\}
$$

Here we recall that

$$
V_{\omega, k}=\nabla \cdot\left(\sum_{\gamma \in C_{k} \cap \mathbb{Z}^{d}} \omega_{\gamma} \sum_{\beta \in(2 k+1) \mathbb{Z}^{d}} \rho^{0}(\cdot-\gamma-\beta)\right) \cdot \nabla .
$$

Then $\mathbf{E}_{\varepsilon, \omega}$ has a probability at least $1-\mathbb{P}_{\varepsilon}$ where $\mathbb{P}_{\varepsilon}$ satisfies

$$
\limsup _{\varepsilon \rightarrow 0^{+}} \frac{\log \left|\log \left(\mathbb{P}_{\varepsilon}\right)\right|}{\log \varepsilon} \leq-\frac{d}{\nu-d}
$$

Using the fact that if for some $C>0$ (depending only on $\delta$ and $\rho^{*}$ ), $V_{\omega, k} \geq$ $-C \varepsilon \Delta$, then the spectrum of $H_{\omega, k}$ does not intersect $\left(E_{+}, E_{+}+\varepsilon\right)$ for $\varepsilon$ small. One computes

$$
\begin{aligned}
\mathbb{E}\left(N_{\omega, k}\left(E_{+}+\varepsilon\right)-N_{\omega, k}\left(E_{+}\right)\right) & =\mathbb{E}\left(\left[N_{\omega, k}\left(E_{+}+\varepsilon\right)-N_{\omega, k}\left(E_{+}\right)\right]_{\left.\mathbf{1}_{\left\{\omega ; V_{\omega, k} \geq-C \varepsilon \Delta\right\}}\right)}\right) \\
& +\mathbb{E}\left(\left[N_{\omega, k}\left(E_{+}+\varepsilon\right)-N_{\omega, k}\left(E_{+}\right)\right]_{\left\{\omega ; V_{\omega, k}<-C \varepsilon \Delta\right\}}\right) \\
& \leq C \mathbb{P}\left(\left\{\omega ; V_{\omega, k}<-C \varepsilon \Delta\right\}\right) \\
& =C\left(1-\mathbb{P}\left(\mathbf{E}_{C \cdot \varepsilon, \omega}\right)\right)=C \mathbb{P}_{C \cdot \varepsilon} .
\end{aligned}
$$

Here, we have used the fact that $N_{\omega, k}$ is bounded, locally uniformly in energy, uniformly in $\omega, k$ by $C$. Taking (3.32) into account, we end the proof of (3.31) and so (3.29) is proved.

Now one deals with the proof of (3.30). We recall that here one supposes once more that $n$ is non-degenerate. The idea is similar to the short range case (we will compare the IDS of our operator to the IDS of another one) and we will follow and use results given in [11, 13]. Let $N^{a}$ be the IDS of the follwoing Anderson discrete operator acting on $l^{2}\left(\mathbb{Z}^{d}\right)$ :

$$
\left(H_{\omega}^{a} u\right)(\alpha)=E_{+} \cdot u(\alpha)+\sum_{|\alpha-\beta|=1}(u(\alpha)-u(\beta))+\left(V_{\omega}^{a} u\right)(\alpha) .
$$

Here $V_{\omega}^{a}$ the diagonal infinite matrix with $v_{\alpha}(\omega)=\sum_{\beta \in \mathbb{Z}^{d}} \omega_{\beta}(1+|\alpha-\beta|)^{-\nu}$ for the $\alpha^{\text {th }}$ diagonal coefficient. 
For $k \in \mathbb{N}^{*}$ and $u \in l^{2}\left(\mathbb{Z}^{d} \cap C_{k}\right)$, let $H_{0}^{k}, V_{\omega}^{k}$ and $H_{\omega}^{k}$ be the following discrete operators

$$
\left(H_{0}^{k} u\right)(\alpha)=E_{+} \cdot u(\alpha)+\sum_{|\alpha-\beta|=1, \beta \in C_{k}}(u(\alpha)-u(\beta)), \quad\left(V_{\omega}^{k} u\right)(\alpha)=v_{\alpha}(\omega) u(\alpha)
$$

and

$$
H_{\omega}^{k}=H_{0}^{k}+V_{\omega}^{k}
$$

Let $N_{k}^{a}$ be the IDS of $H_{\omega}^{k}$ defined by:

$$
N_{k}^{a}(E)=\frac{1}{(2 k+1)^{d}} \cdot \mathbb{E}\left(\sharp\left\{\text { eigenvalues of } H_{\omega}^{k} \text { less or equal to } E\right\}\right) .
$$

From [11, 13], we know that for a good choice of $k$, the IDS at energy $E$ is quite well approximated by the probability to find a state energy less than $E$. Precisely we have the following relation:

$$
N^{a}(E) \leq N_{k}^{a}(E) \leq C \cdot \mathbb{P}_{k}(E)
$$

Here

$$
\mathbb{P}_{k}(E)=\mathbb{P}\left(\left\{H_{\omega}^{k} \text { admits at least an eigenvalue less than } E\right\}\right) .
$$

To estimate this probability one proceeds as previously and lower bound $H_{\omega}^{k}$ by $H_{\widetilde{\omega}}^{k}$; obtained for $\delta>0$ by changing $\omega_{\gamma}$ by $\widetilde{\omega}_{\gamma}=\omega_{\gamma}$ if $\omega_{\gamma} \leq \delta$ and $\widetilde{\omega}_{\gamma}=\delta$ if not. So if we denote the IDS of $H_{\widetilde{\omega}}^{k}$ by $\widetilde{N}_{k}^{a}$ then we have,

$$
N_{k}^{a}(E) \leq \tilde{N}_{k}^{a}(E) .
$$

One takes $k=c\left(E-E_{+}\right)^{-\frac{1}{2}}$ and $\delta=\left(E-E_{+}\right) / c$ positives. For a good choice of $c$ and thus of $\delta$, and $E$ in a neighborhood of $E_{+}$, we get

$$
\mathbb{P}_{k}(E) \leq \mathbb{P}\left(\left\{\widetilde{\omega} ; \frac{1}{(2 k+1)^{d}} \sum_{|\gamma| \leq k} v_{\gamma}(\widetilde{\omega}) \leq \delta / K\right\}\right)
$$

Now we have to estimate the last probability. Let $0<\alpha<1$, for some $C>1$ we have:

$$
\frac{1}{(2 k+1)^{d}} \sum_{|\gamma| \leq k} v_{\gamma}(\widetilde{\omega})=\frac{1}{(2 k+1)^{d}} \sum_{\beta \in \mathbb{Z}^{d}} \widetilde{\omega}_{\beta}\left(\sum_{|\gamma| \leq k}(1+|\gamma-\beta|)^{-\nu}\right) .
$$




$$
\geq \frac{1}{C(2 k+1)^{d}} \sum_{|\beta| \leq k} \widetilde{\omega}_{\beta}+\frac{1}{C} \sum_{k<|\beta| \leq \delta^{-(\nu-d)(1-\alpha)}} \widetilde{\omega}_{\beta}(1+|\beta|+k)^{-\nu} .
$$

Thus

$$
\begin{aligned}
& \mathbb{P}\left(\left\{\widetilde{\omega} ; \frac{1}{(2 k+1)^{d}} \sum_{|\gamma| \leq k} v_{\gamma}(\widetilde{\omega}) \leq \delta / K\right\}\right) \leq \\
& \mathbb{P}\left(\left\{\widetilde{\omega} ; \frac{1}{C(2 k+1)^{d}} \sum_{|\beta| \leq k} \widetilde{\omega}_{\beta} \leq \delta / K \text { and } \frac{1}{C} \sum_{k<|\beta| \leq \delta^{-(\nu-d)(1-\alpha)}} \widetilde{\omega}_{\beta}(1+|\beta|+k)^{-\nu} \leq \delta / K\right\}\right) .
\end{aligned}
$$

As the random variables are i.i.d we get for

$$
\mathbb{P}_{1}=\mathbb{P}\left(\left\{\widetilde{\omega} ; \frac{1}{C(2 k+1)^{d}} \sum_{|\beta| \leq k} \widetilde{\omega}_{\beta} \leq \delta / K\right\}\right)
$$

and

$$
\mathbb{P}_{2}=\mathbb{P}\left(\left\{\widetilde{\omega} ; \frac{1}{C} \sum_{k<|\beta| \leq \delta^{-(\nu-d)(1-\alpha)}} \widetilde{\omega}_{\beta}(1+|\beta|+k)^{-\nu} \leq \delta / K\right\}\right),
$$

we have

$$
\mathbb{P}\left(\left\{\widetilde{\omega} ; \frac{1}{(2 k+1)^{d}} \sum_{|\gamma| \leq k} v_{\gamma}(\widetilde{\omega}) \leq \delta / K\right\}\right) \leq \mathbb{P}_{1} \cdot \mathbb{P}_{2}
$$

The estimation of $\mathbb{P}_{1}$ and $\mathbb{P}_{2}$ is based on large deviation results [2]. Briefly the idea is the following. Let $t>0$. Using the Markov inequality one estimates

$$
\mathbb{P}_{1} \leq \mathbb{E}\left(e^{t\left(\delta / K-\frac{1}{(2 k+1)^{d}} \sum_{|\beta| \leq k} \tilde{\omega}_{\gamma}\right)}\right) .
$$

As the random variables are i.i.d one gets

$$
\begin{aligned}
\left.\mathbb{E}\left(e^{t\left(\delta / K-\frac{1}{(2 k+1)^{d}} \sum_{|\beta| \leq k} \widetilde{\omega}_{\gamma}\right.}\right)\right) & =e^{t \delta / K} \prod_{|\beta| \leq k} \mathbb{E}\left(e^{-t \widetilde{\omega}_{\beta} /(2 k+1)^{d}}\right) \\
& =e^{t \delta / K} e^{(2 k)^{d} \log \mathbb{E}\left(e^{-t \tilde{\omega}_{0} /(2 k+1)^{d}}\right)}
\end{aligned}
$$

For $k$ big enough using Taylor expansion of $e^{t}$, we get that

$$
\mathbb{E}\left(e^{-t \frac{\tilde{\omega}_{0}}{(2 k)^{d}}}\right)=1-\frac{t \mathbb{E}\left(\widetilde{\omega}_{0}\right)}{(2 k+1)^{d}}+o\left(\frac{t^{2}}{(2 k+1)^{2 d}}\right) .
$$


So we get

$$
\mathbb{P}_{1} \leq e^{t \delta / K} e^{(2 k)^{d} \log \left(1-\frac{t \mathbb{E}\left(\tilde{\omega}_{0}=0\right)}{(2 k+1)^{d}}+o\left(\frac{t^{2}}{(2 k+1)^{2 d}}\right)\right)} .
$$

As $\mathbb{E}\left(\widetilde{\omega}_{0}\right)>0$ for $k$ big enough and $t>0$ well chosen we get that there exists $C>0$ such that

$$
\mathbb{P}_{1} \leq e^{\left((2 k)^{d} \log \left(\mathbb{P}\left\{\widetilde{\omega}_{0}=0\right\}\right) / C\right.} .
$$

The same computation as above gives

$$
\mathbb{P}_{2} \leq e^{-\left(k^{d}\left|\log \left(\mathbb{P}\left\{\tilde{\omega}_{0} \leq \delta\right\}\right)\right|+\delta^{-d /(\nu-d)}\right) / C}
$$

So, we get that:

$$
\log N^{a}(E) \leq-\left(k^{d}\left|\log \mathbb{P}\left(\left\{\widetilde{\omega}_{0}=0\right\}\right)\right|+\left|\log \left(\mathbb{P}\left(\widetilde{\omega}_{0}=0\right)\right)\right|+\delta^{-d /(\nu-d)}\right) / C .
$$

Now, for $k=c\left(E-E_{+}\right)^{-\frac{1}{2}}$ and $\delta=\left(E-E_{+}\right) / c$ we get 3.30 for $N^{a}$.

As $n$ is non-degenerate we get that (see the short range case and [9]) $H_{\omega}^{a}$ is unitarly equivalent by means of the Fourier transformation to some operator which lower bound $H_{\omega}^{0}$. This yelds (3.30) for $N$.

Acknowledgements. I would like to thank professor Mabrouk Ben Ammar and my colleague Adel Khalfallah for theirs supports.

\section{References}

[1] A. Figotin and A. Klein: Localization of Classical Waves I :Acoustic Waves. Commun. Math. Phys. 180 (1996) p 439-482

[2] A. Dembo and O. Zeitouni. Large deviation and applications. Jones and Bartlett Publishers, Boston, 1992.

[3] W. Kirsch and F. Martinelli: On the the Spectrum Of Schrödinger operators with a Random Potential. Commun. Math. Phys. 85 (1982) p 329-350.

[4] W. Kirsch: Random Schrödinger operators A Course Lecture Notes In Phys. 345 (1989)Springer-Verlag, Berlin p 264-370. 
[5] F. Klopp: Internal Lifshitz Tails For Random Perturbations Of Periodic Schrödinger Operators. Duke Math. Jour.

[6] F. Klopp: Weak disorder localization and Lifshitz Tails:continuous hamiltonian. Anna. I.A.P 3 (2002) N 4 p 711-735.

[7] F. Klopp: Internal Lifshitz Tails For long range single site potentials. Jour. Math. Phys. 43 (2002) Nº 6 p 2948-2958.

Commu. Math. Phys.(1999) 206 p 57-103.

[8] H. Najar: Asymptotique de la densité d'états intégrée des opérateurs acoustiques aléatoires. C. R. Acad. Sci. Paris, 333 I, p 191-194 (2001).

[9] H. Najar: Lifshitz tails for random acoustic operators. Jour. Math. Phys. $44 \mathrm{~N}^{\circ} 4$ p1842-1867 (2003).

[10] H. Najar: Asymptotic behavior of the integrated density of states of acoustic operator with long range random perturbations. Jour. Stat. Phys. $115 \mathrm{~N}^{\circ} 4$ p 977-955 (2003).

[11] L. Pastur and A. Figotin: Spectra of Random and Almost-Periodic Operators. Springer-Verlag.

[12] M. Reed and B. Simon: Methods of Modern Mathematical vol IV: Analysis of Operators. New York: Academic Press 1978.

[13] B. Simon: Lifshitz tails for the Anderson models. Jour. Stat. Phy. 38 p 65-76 (1985)

[14] P. Stollmann: Caught by Disorder Bounded States in random Media. Birkhäuser.

[15] I. Veselić: Localization for random perturbation of periodic Schrödinger operators with regular Floquet eigenvalues. Technical report Univ. Bochum. 